\title{
Carboxamide Spleen Tyrosine Kinase (Syk) Inhibitors: Leveraging Ground State Interactions To Accelerate Optimization
}

\author{
J. Michael Ellis,* Michael D. Altman,* Brandon Cash, Andrew M. Haidle, Rachel L. Kubiak, \\ Matthew L. Maddess, Youwei Yan, and Alan B. Northrup \\ Department of Discovery Chemistry, Merck \& Co., Inc., 33 Avenue Louis Pasteur, Boston, Massachusetts 02115, United States
}

\section{Supporting Information}

ABSTRACT: Optimization of a series of highly potent and kinome selective carbon-linked carboxamide spleen tyrosine kinase (Syk) inhibitors with favorable drug-like properties is described. A pervasive Ames liability in an analogous nitrogen-linked carboxamide series was obviated by replacement with a carbon-linked moiety. Initial efforts lacked on-target potency, likely due to strain induced between the hinge binding amide and solvent front heterocycle. Consideration of ground state and bound state energetics allowed rapid realization of improved solvent front substituents affording subnanomolar Syk potency and high kinome selectivity. These molecules were also devoid of mutagenicity risk as assessed via the Ames test using the TA97a Salmonella strain.

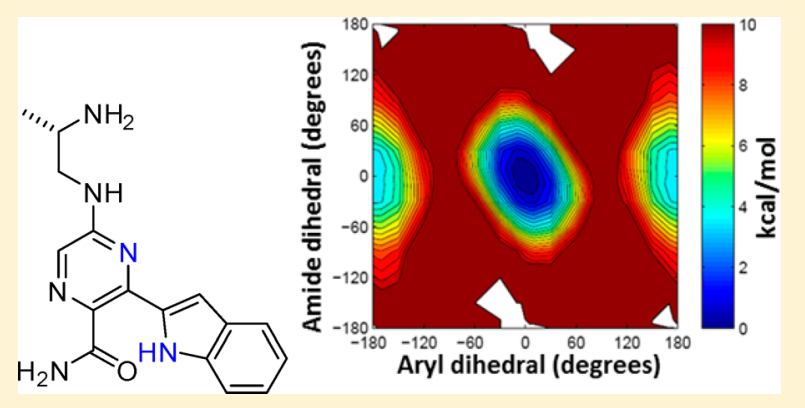

KEYWORDS: Spleen tyrosine kinase, intramolecular hydrogen bond, structure-based drug design

$\mathrm{T}$ he advancement of kinase inhibitors as oncology therapeutics has resulted in approval of a number of effective drugs over the past two decades. Recently, efforts have been directed toward the treatment of autoimmune disorders, particularly rheumatoid arthritis (RA), via kinase inhibition. RA is a chronic inflammatory disease that presents with pathophysiology including synovial hyperplasia, inflammation, and joint destruction. RA is characterized by up-regulation of cytokines and chemokines ultimately leading to inflammation. Treatment paradigms have consisted of palliative therapies to mitigate symptoms including pain and disease-modifying antirheumatic drugs (DMARDs) or biologics with the goal to slow or stop disease progression. Existing therapies are not universally effective for all RA patients, underscoring the need for additional treatment options.

Spleen tyrosine kinase (Syk) is a well-studied enzyme that plays a critical role in immune signaling via $F_{c}$ and $B$ cell receptors (BCR). ${ }^{1}$ Preclinical studies demonstrated that pharmacological modulation of Syk (R788 (1), PRT-062607 (2), RO9021 (3), and GS-9973 (4) is efficacious in preclinical RA models (Figure 1). ${ }^{2-5}$ Further, poorly selective Syk

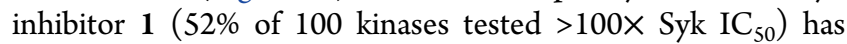
demonstrated efficacy in human clinical trials; however, dosing was limited by observations of hypertension and diarrhea. ${ }^{6}$ We aimed to develop a selective Syk inhibitor that would obviate the clinical side effects and could be useful for autoimmune and oncological disorders. ${ }^{7-11}$ As previously described, ${ }^{12}$ a picolinamide series of Syk inhibitors we developed was halted by mutagenicity risk as indicated by positive results in the Ames assay. Described herein are the design considerations employed<smiles>COc1cc(Nc2ncc(F)c(Nc3ccc4c(n3)N(COP(=O)(O)O)C(=O)C(C)(C)O4)n2)cc(OC)c1OC</smiles>

Fostamatinib, R788 (1)

PRT-062607 (2)<smiles>Cc1ccc(Nc2cc(N[C@H]3CCCC[C@H]3N)nnc2C(N)=O)nc1C</smiles>

RO-9021 (3)<smiles>c1cn2cc(-c3ccc4cn[nH]c4c3)nc(Nc3ccc(N4CCOCC4)cc3)c2n1</smiles>

Entospletinib, GS-9973 (4)
Figure 1. Diverse published Syk inhibitors.

to overcome this liability via a scaffold modification affording novel Syk inhibitors consistent with our objective.

Picolinamide 5 was designed via analysis of X-ray crystallographic data with a focus on ligand binding efficiency and ensuring favorable physicochemical properties (Figure 2).

Received: September 5, 2016

Accepted: October 7, 2016

Published: October 7, 2016 
<smiles>Cc1cc(C)nc(Nc2cc(N[C@@H]3CCCC[C@H]3N)cnc2C(N)=O)c1</smiles>

Figure 2. Lead molecule picolinamide $\mathbf{5}$.

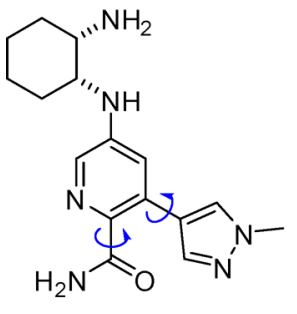

6<smiles>C[C@H](N)CNc1cnc(C(N)=O)c(-c2cnn(C)c2)n1</smiles><smiles>C[C@H](N)CNc1cnc(C(N)=O)c(-c2cc3ccccc3[nH]2)n1</smiles>
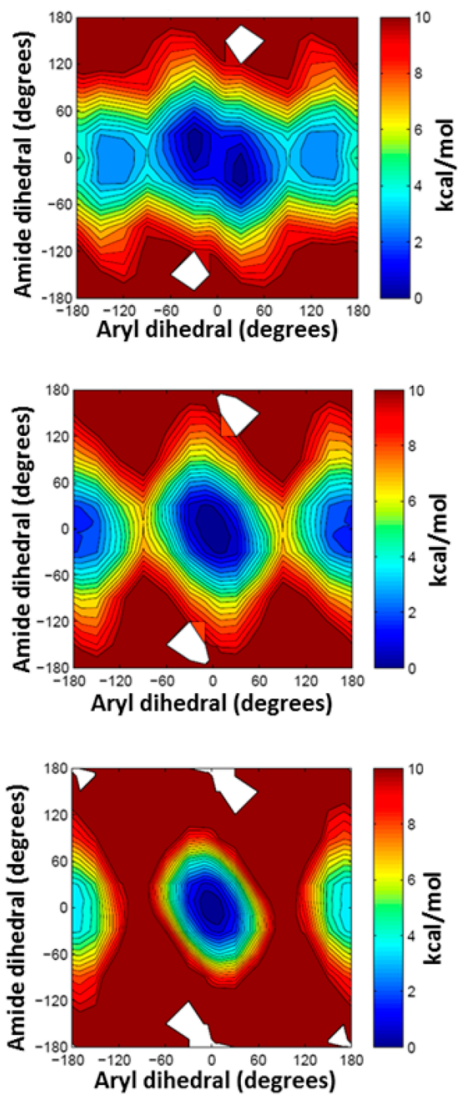

Figure 3. Initial $C$-linked carboxamides and predicted quantum mechanical potential energy landscape for rotation about the aryl and amide dihedral angles. $(0,0)$ represents the presumed planar bioactive conformation in the orientation as drawn. White regions represent configurations with severe intramolecular clashes, and thus, no energy was computed. This figure was prepared with MATLAB.<smiles>[R]c1cnc(C(N)=O)c(-c2c(O)nn(C)c2[14CH2])c1[Y]</smiles>

9 versus<smiles>[R]#Cc1ncc(C(N)=O)c(-c2nn(C)nc2O)n1</smiles>

10
Figure 4. Rationale for picolinamide and pyrazinecarboxamide ground state dihedral angles and resultant intrinsic potencies.

Carboxamide 5 possessed desirable intrinsic (Syk $\mathrm{IC}_{50}=60$ $\mathrm{pM}$ ) and human whole blood potency (hWB CysLT $\mathrm{IC}_{50}=58$ $\mathrm{nM}$ ). Additionally, kinome selectivity was high (99\% of 265 kinases tested $>100 \times$ Syk $\left.\mathrm{IC}_{50}\right),{ }^{13}$ and the oral pharmacokinetic profile (rat plasma $\mathrm{Cl}_{\mathrm{p}}\left(\mathrm{Cl}_{\mathrm{u}}\right)=33(710) \mathrm{mL} / \mathrm{min} / \mathrm{kg}$, rat $T_{1 / 2}=$ $2.7 \mathrm{~h}, \mathrm{~F}=19 \%)$ enabled testing in the rat collagen-induced

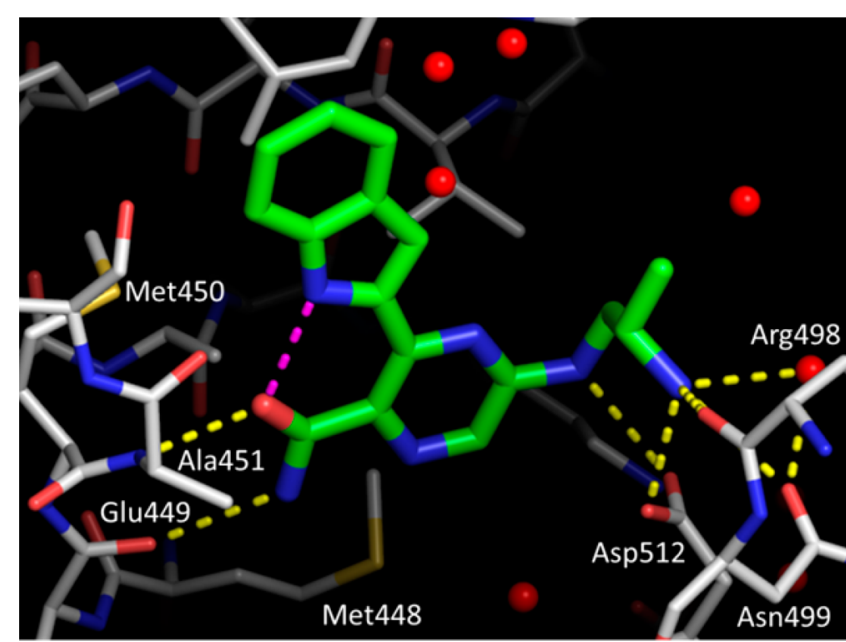

Figure 5. Binding site view of the crystal structure of 8 bound to the human Syk kinase domain. The carbon atoms of Syk protein are shown in white, and carbon atoms of $\mathbf{8}$ are in green. Modeled water molecules are shown as red spheres, an intramolecular hydrogen bond in $\mathbf{8}$ is shown in magenta dashes, and polar interactions between $\mathbf{8}$ and protein are shown as yellow dashes. This figure was prepared with PyMOL.

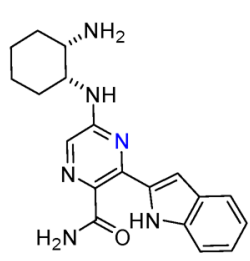

11

Syk $\mathrm{IC}_{60}: 0.5 \mathrm{nM}$

hWB CysLT IC $50: 130 \mathrm{nM}$

Kinases >100X: $99 \%$ (100)

Rat $\mathrm{Cl}_{\mathrm{p}}\left(\mathrm{Cl}_{\mathrm{u}}\right): 44(255) \mathrm{mL} / \mathrm{min} / \mathrm{kg}$

Rat $\mathbf{T}_{1 / 2}: 2.8 \mathrm{~h}, 19 \% \mathrm{~F}$

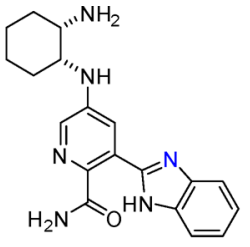

12

Syk $\mathrm{IC}_{50}: 7 \mathrm{nM}$

hWB CysLT IC $50: 93$ nM

Kinases >100X: 97\% (100)

Rat $\mathrm{CI}_{\mathrm{p}}\left(\mathrm{Cl}_{\mathbf{u}}\right): 134(277) \mathrm{mL} / \mathrm{min} / \mathrm{kg}$

Rat $\mathrm{T}_{1 / 2}: 4.8 \mathrm{~h}$
Figure 6. Advanced C-linked carboxamides.

arthritis model, where the compound demonstrated therapeutic efficacy at doses as low as $3 \mathrm{mg} / \mathrm{kg}$ QD $\left(C_{\max }=0.2 \mu \mathrm{M}, C_{\min }=\right.$ $0.007 \mu \mathrm{M})$. However, this molecule, and this series of diaminopicolinamides, was plagued with positive results in the Ames test using TA97a Salmonella strain. Since mutation in this strain often reflects intercalation of the test molecule into DNA, we developed a docking model leveraging DNA cocrystals with acridine. Based upon this model, an approach we pursued to ameliorate this activity involved changing the two-dimensional shape of how the molecules are presented, aimed at disrupting the favorable interactions with DNA.

Our initial foray into changing two-dimensional shape focused on altering the vector at which the "solvent front" group was presented; this strategy involved carbon-linking to replace the previous nitrogen-linked moiety. To this end, picolinamide 6 was quickly prepared via Suzuki coupling ${ }^{14}$ of available intermediates to replace the aminoheterocycle with a C-linked 3-methylpyrazole analogue (Figure 3). However, picolinamide 6 was inactive in our enzymatic assay $\left(\right.$ Syk $\mathrm{IC}_{50}$ $>10 \mu \mathrm{M}, 9 \%$ inhibition at $10 \mu \mathrm{M})$. We hypothesized that the 3methylpyrazole may introduce torsional strain with the carbonyl oxygen of the carboxamide, disfavoring the planar geometry desired for efficient interaction with the hinge 
Table 1. Additional C-Linked Carboxamide SAR

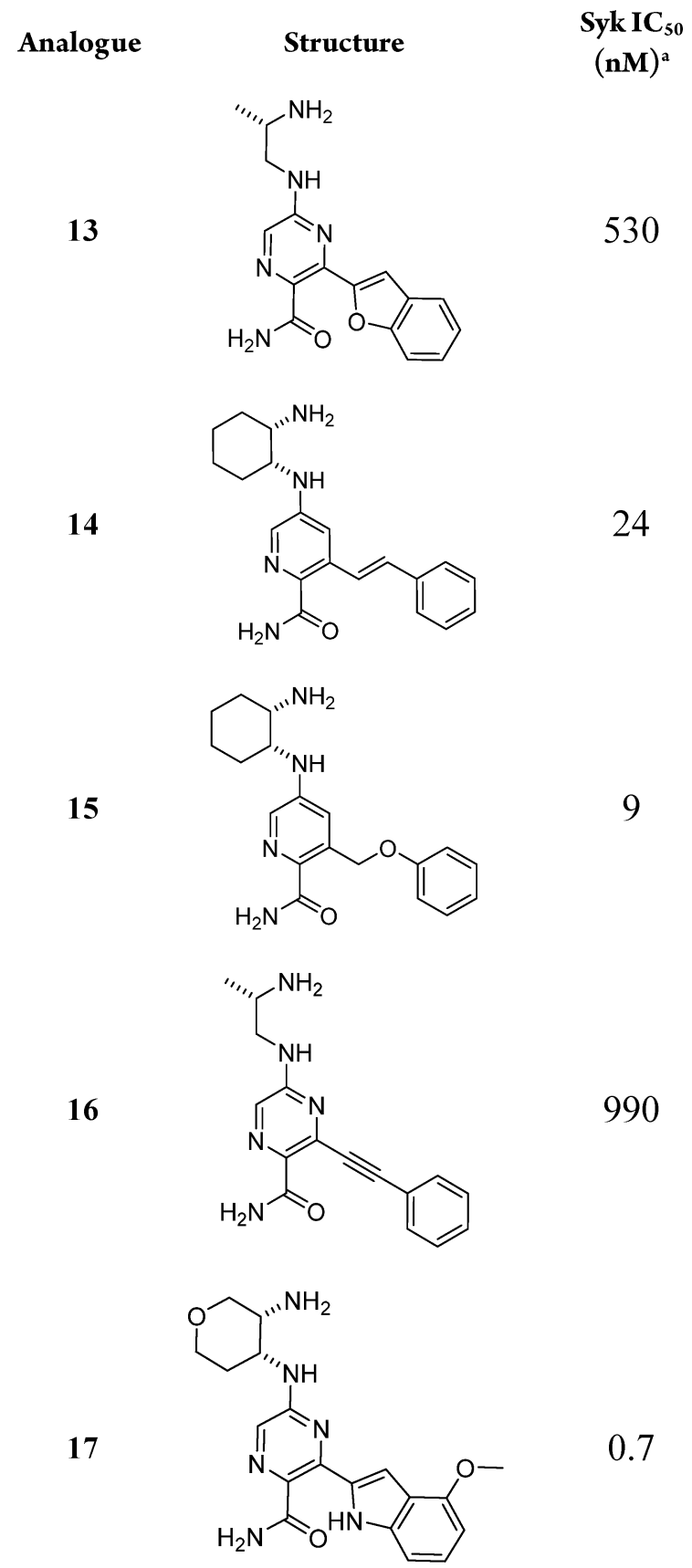

${ }^{a}$ See Supporting Information for details.

domain of Syk, and kinases in general. To probe this theory, we pursued computational quantum mechanical approaches to estimate the lowest energy dihedral angles about these two substituents and the energetic penalty required to achieve a fully flat conformation (amide dihedral $=0^{\circ}$ and aryl solvent front dihedral $=0^{\circ}$ ) for binding. ${ }^{15}$ For picolinamide 6, calculations suggest that the preferred ground state conformation positions the two critical substituents $\sim 30^{\circ}$ and $-30^{\circ}$ out of plane, resulting in an estimated penalty of $2-4$ $\mathrm{kcal} / \mathrm{mol}$ to adopt the planar bound-state conformation, which is reflected in the lack of potency for this analogue.

Considering rational structural modifications that could be implemented to further favor the flat ground state conformation, minimizing the motion and associated energetic penalty required to achieve the flat bioactive state, calculations were executed to examine the roles of different heteroatom substituted cores and solvent fronts. Among those surveyed, the pyrazinecarboxamide core was predicted to favor a smaller dihedral angle, and corresponding lower energetic penalty ( $0-2$ $\mathrm{kcal} / \mathrm{mol}$ ) than the picolinamide. In the picolinamide 9 , there is likely increased torsional strain resulting from the two $\mathrm{CHs}$ in close proximity compared to the pyrazine 10, where this strain is removed and the conformation may be reinforced by an additional intramolecular hydrogen bond (Figure 4). Pyrazinecarboxamide 7 was prepared to test this hypothesis and showed improved potency $\left(\right.$ Syk $\left.\mathrm{IC}_{50}=1.6 \mu \mathrm{M}\right)$. It is worth noting that the cyclohexyldiamine generally introduces a $\sim 10 \times$ potency enhancement versus the propanyldiamine, though this matched pair was never made with the methypyrazole solvent front.

Hoping to parlay the potency gain achieved moving from the picolinamide to the pyrazinecarboxamide with a solvent front group predicted to further favor a planar disposition, indole $\mathbf{8}$ was synthesized. ${ }^{16}$ Calculations suggested that the indole substituted pyrazinecarboxamide should strongly favor a flat ground state conformation, resulting in a $0-0.5 \mathrm{kcal} / \mathrm{mol}$ energetic penalty to adopt the bound conformation. In addition, the energy minimum at the presumed bound conformation is predicted to become much deeper than alternative states, which likely reduces entropic penalties as well. This is likely influenced by an intramolecular hydrogen bond between the more polarized $\mathrm{NH}$ of the indole and the carbonyl of the carboxamide. Gratifyingly, indole 8's doubly stabilized planarity afforded a significantly improved intrinsic potency (Syk $\mathrm{IC}_{50}=5 \mathrm{nM}$ ). Interestingly, the predicted ground-state geometry for $\mathbf{8}$ suggests a pseudo 7-membered ring where the bond angle increases to accommodate an intramolecular hydrogen bond between the indole $\mathrm{NH}$ and the carboxamide carbonyl (Figure S1). ${ }^{15}$

In order to further understand the binding interactions of these compounds, as well as interrogate the computational predictions, we solved the X-ray crystal structure of compound 8 bound to the kinase domain of human Syk (Figure 5). Similar to other carboxamide-based Syk inhibitors, ${ }^{17-20} 8$ binds to the hinge region via bidentate hydrogen bonding to the backbone of Glu449 and Ala451. The indole region extends toward solvent and packs against the side chain of Met450 at the top of the hinge, while $\mathrm{NH}$ groups of the diamine region form interactions with the catalytic Asp512 and surrounding backbone carbonyls from Arg498 and Asn499. As predicted, the core is planar with a pseudo 7 -membered ring geometry stabilized by an intramolecular hydrogen bond between the indole $\mathrm{NH}$ and the carboxamide oxygen with a short $\mathrm{N}-\mathrm{O}$ distance of $2.6 \AA$ (Figure 5, magenta dashed line).

Seeking to further optimize the $C$-linked carboxamides, introduction of the cyclohexyldiamine moiety to afford pyrazinecarboxamide $\mathbf{1 1}$ delivered the expected potency enhancement (Syk $\left.\mathrm{IC}_{50}=500 \mathrm{pM}\right)$ approaching the previously achieved intrinsic activity of the $N$-linked picolinamide $\mathbf{5}$. Further, indole 11 featured excellent kinase selectivity (99\% of

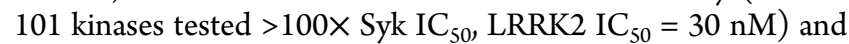
human whole blood potency (hWB CysLT $\mathrm{IC}_{50}=130 \mathrm{nM}$ ) with a favorable pharmacokinetic profile in rat $\mathrm{Cl}_{\mathrm{p}}\left(\mathrm{Cl}_{\mathrm{u}}\right)=44$ (255) $\mathrm{mL} / \mathrm{min} / \mathrm{kg}$, rat $T_{1 / 2}=2.8 \mathrm{~h}, \mathrm{~F}=19 \%$ ) (Figure 6). As previously described, all molecules tested from the $C$-linked carboxamides were negative in the mutagenicity assay in strain TA97a Salmonella with rat S9 up to testable concentrations. ${ }^{12}$ Additional data to support the effectiveness of the shape change 
in ablating interaction with DNA were gathered via consistent results in a UV-vis perturbation assay and a DNA unwinding assay. We subsequently examined a nitrogen transposition of pyrazinecarboxamide 11 to afford benzimidazole 12. Interestingly, picolinamide 12 showed a loss in intrinsic potency (Syk $\left.\mathrm{IC}_{50}=7 \mathrm{nM}\right)$ but a maintained selectivity (97\% of 101 kinases tested $>100 \times$ Syk IC $_{50}$, LRRK2 IC IC $_{50} 130 \mathrm{nM}$, CHK2 IC $\mathrm{I}_{50}=$ $160 \mathrm{nM}$, TSSK3 $\left.\mathrm{IC}_{50}=420 \mathrm{nM}\right)$, human whole blood potency $\left(\mathrm{hWB}\right.$ CysLT $\left.\mathrm{IC}_{50}=93 \mathrm{nM}\right)$, and rat pharmacokinetic $\left(\mathrm{Cl}_{\mathrm{p}}\right.$ $\left(\mathrm{Cl}_{\mathrm{u}}\right)=134(277) \mathrm{mL} / \mathrm{min} / \mathrm{kg}$, rat $\left.T_{1 / 2}=4.8 \mathrm{~h}\right)$ profile.

Encouraged by the successes of exploiting intramolecular hydrogen bonds, we investigated the use of weaker $H$-bond donors and other configurations predicted to maintain a planar geometry. Benzofuran 13 lost $\sim 100 \times$ potency (Syk $\mathrm{IC}_{50}=530$ $\mathrm{nM}$ ) versus its indole analogue $\mathbf{8}$. Styrene 14 had comparable intrinsic affinity, $\sim 3 \times$ loss $\left(\right.$ Syk $\mathrm{IC}_{50}=24 \mathrm{nM}$ ) versus the corresponding benzimidazole 12. Aryl ether 15, interestingly predicted to prefer a planar configuration, affords an equipotent inhibitor $($ Syk IC50 $=9 \mathrm{nM})$ to benzimidazole 12. Alkyne 16 was readily prepared but demonstrated a substantial potency loss $\left(\right.$ Syk $\left.\mathrm{IC}_{50}=990 \mathrm{nM}\right)$, likely due to negative interaction of the phenyl moiety with the hinge region of Syk (Table 1).

The combined efforts of this scaffold redesign ultimately delivered pyrazinecarboxamide $17,^{21}$ which possessed the overall profile we were seeking $\left(\right.$ Syk $\left.\mathrm{IC}_{50}=700 \mathrm{pM}\right)$ including high selectivity ( $99 \%$ of kinases $>100 \times, 101$ kinases tested, LRRK2 $\mathrm{IC}_{50}=33 \mathrm{nM}$ ), potent cell functional activity (hWB CysLT $\mathrm{IC}_{50}=62 \mathrm{nM}$ ), and a favorable pharmacokinetic profile in preclinical species (rat plasma $\mathrm{Cl}_{\mathrm{p}}\left(\mathrm{Cl}_{\mathrm{u}}\right)=25(100) \mathrm{mL} /$ $\mathrm{min} / \mathrm{kg}$, rat $T_{1 / 2}=3.5 \mathrm{~h}, 29 \% \mathrm{~F}$ ). Further, indole 17 posed low risk for ion channel activity and DNA interaction as assessed by preclinical safety assays ( $\mathrm{hERG} \mathrm{IC}_{50}=22 \mu \mathrm{M}$ and UV-vis = negative). In summary, careful consideration of designs to bias toward the bioactive conformation delivered alternative chemotypes that maintained favorable drug-like properties while overcoming several safety issues.

\section{ASSOCIATED CONTENT}

\section{S Supporting Information}

The Supporting Information is available free of charge on the ACS Publications website at DOI: 10.1021/acsmedchemlett.6b00353.

Experimental procedures for synthesis of final compounds and intermediates, descriptions of assays performed, computational methods with additional results, and crystallographic details (PDF)

\section{Accession Codes}

The complex between the human Syk kinase domain and compound $\mathbf{8}$ has been deposited in the Protein Data Bank (PDB) with accession code 5TIU.

\section{AUTHOR INFORMATION}

\section{Corresponding Authors}

*Phone: +1-617-992-3144. E-mail: michael_ellis@merck.com. *Phone: +1-617-992-3077. E-mail: michael_altman@merck. com.

\section{Author Contributions}

All authors have given approval to the final version of the manuscript.

\section{Notes}

The authors declare the following competing financial interest(s): All authors are employed by Merck \& Co., Inc., who supported and funded these research efforts.

\section{ACKNOWLEDGMENTS}

We would like to acknowledge Jaren Arbanas and Uwe Mueller for routine in vitro pharmacology support, and Bruce Adams and Bridget Becker for NMR structural support.

\section{ABBREVIATIONS}

Syk, spleen tyrosine kinase; RA, rheumatoid arthritis; DMARD, disease-modifying antirheumatic drug; Fc, fragment, crystallizable; BCR, B cell receptor; Syk $\mathrm{IC}_{50}$, recombinant human Syk $\mathrm{IC}_{50}$; hWB, human whole blood; PEG, polyethylene glycol

\section{REFERENCES}

(1) Geahlen, R. L. Getting Syk: spleen tyrosine kinase as a therapeutic target. Trends Pharmacol. Sci. 2014, 35, 414-422.

(2) Pine, P. R.; Chang, B.; Schoettler, N.; Banquerigo, M. L.; Wang, S.; Lau, A.; Zhao, F.; Grossbard, E. B.; Payan, D. G.; Brahn, E. Inflammation and bone erosion are suppressed in models of rheumatoid arthritis following treatment with a novel Syk inhibitor. Clin. Immunol. 2007, 124, 244-257.

(3) Coffey, G.; DeGuzman, F.; Inagaki, M.; Pak, Y.; Delaney, S. M.; Ives, D.; Betz, A.; Jia, Z. J.; Pandey, A.; Baker, D.; Hollenbach, S. J.; Phillips, D. R.; Sinha, U. Specific inhibition of spleen tyrosine kinase suppresses leukocyte immune function and inflammation in animal models of rheumatoid arthritis. J. Pharmacol. Exp. Ther. 2012, 340, $350-359$.

(4) Liao, C.; Hsu, J.; Kim, Y.; Hu, D.-Q.; Xu, D.; Zhang, J.; Pashine, A.; Menke, J.; Whittard, T.; Romero, N.; Truitt, T.; Slade, M.; Lukacs, C.; Hermann, J.; Zhou, M.; Lucas, M.; Narula, S.; DeMartino, J.; Tan, S.-L. Selective inhibition of spleen tyrosine kinase (SYK) with a novel orally bioavailable small molecule inhibitor, RO9021, impinges on various innate and adaptive immune responses: implications for SYK inhibitors in autoimmune disease therapy. Arthritis Res. Ther. 2013, 15, R146.

(5) Currie, K. S.; Kropf, J. E.; Lee, T.; Blomgren, P.; Xu, J.; Zhao, Z.; Gallion, S.; Whitney, J. A.; Maclin, D.; Lansdon, E. B.; Maciejewski, P.; Rossi, A. M.; Rong, H.; Macaluso, J.; Barbosa, J.; Di Paolo, J. A.; Mitchell, S. A. Discovery of GS-9973, a selective and orally efficacious inhibitor of spleen tyrosine kinase. J. Med. Chem. 2014, 57, 38563873.

(6) Weinblatt, M. E.; Kavanaugh, A.; Genovese, M. C.; Jones, D. A.; Musser, T. K.; Grossbard, E. B.; Magilavy, D. B. Effects of fostamatinib (R788), an oral spleen tyrosine kinase inhibitor, on health-related quality of life in patients with active rheumatoid arthritis: analyses of patient-reported outcomes from a randomized, double-blind, placebocontrolled trial. J. Rheumatol. 2013, 40, 369-378.

(7) For the role of VEGF inhibition in hypertension see: Pande, A.; Lombardo, J.; Spangethal, E.; Javle, M. Hypertension secondary to anti-angiogenic therapy: experience with bevacizumab. Anticancer Res. 2007, 27, 3465-3470.

(8) Suljagic, M.; Longo, P. G.; Bennardo, S.; Perlas, E.; Leone, G.; Laurenti, L.; Efremov, D. G. The Syk inhibitor fostamatinib disodium (R788) inhibits tumor growth in the $\mathrm{E} \mu$-TCL1 transgenic mouse model of CLL by blocking antigen-dependent B-cell receptor signaling. Blood 2010, 116, 4894-4905.

(9) Friedberg, J. W.; Sharman, J.; Sweetenham, J.; Johnston, P. B.; Vose, J. M.; Lacasce, A.; Schaefer-Cutillo, J.; De Vos, S.; Sinha, R.; Leonard, J. P.; Cripe, L. D.; Gregory, S. A.; Sterba, M. P.; Lowe, A. M.; Levy, R.; Shipp, M. A. Inhibition of Syk with fostamatinib disodium has significant clinical activity in non-Hodgkin lymphoma and chronic lymphocytic leukemia. Blood 2010, 115, 2578-2585. 
(10) Ulanova, M.; Duta, F.; Puttagunta, L.; Schreiber, A. D.; Befus, A. D. Spleen tyrosine kinase (Syk) as a novel target for allergic asthma and rhinitis. Expert Opin. Ther. Targets 2005, 9, 901-921.

(11) Moy, L. Y.; Jia, Y.; Caniga, M.; Lieber, G.; Gil, M.; Fernandez, X.; Sirkowski, E.; Miller, R.; Alexander, J. P.; Lee, H.-H.; Shin, J. D.; Ellis, J. M.; Chen, H.; Wilhelm, A.; Yu, H.; Vincent, S.; Chapman, R. W.; Kelly, N.; Hickey, E.; Abraham, W. M.; Northrup, A.; Miller, T.; Houshyar, H.; Crackower, M. A. Inhibition of spleen tyrosine kinase attenuates allergen-mediated airway constriction. Am. J. Respir. Cell Mol. Biol. 2013, 49, 1085-1092.

(12) Ellis, J. M.; Altman, M. D.; Bass, A.; Butcher, J. W.; Byford, A. J.; Donofrio, A.; Galloway, S.; Haidle, A. M.; Jewell, J.; Kelly, N.; Leccese, E. K.; Lee, S.; Maddess, M.; Miller, J. R.; Moy, L. Y.; Osimboni, E.; Otte, R. D.; Reddy, M. V.; Spencer, K.; Sun, B.; Vincent, S. H.; Ward, G. J.; Woo, G. H. C.; Yang, C.; Houshyar, H.; Northrup, A. B. Overcoming genotoxicity and ion channel activity: Optimization of selective spleen tyrosine kinase inhibitors. J. Med. Chem. 2015, 58, 1929-1939.

(13) Zap70 IC $\mathrm{IC}_{50}: 5.3 \mathrm{nM}\left(\right.$ Zap70 IC $\mathrm{IC}_{50} /$ Syk $\left.\mathrm{IC}_{50}=90 \mathrm{X}\right)$, which was consistent with the general selectivity index versus this structurally homologous kinase.

(14) Miyaura, N.; Yamada, K.; Suzuki, A. A new stereospecific crosscoupling by the palladium-catalyzed reaction of 1-alkenylboranes with 1-alkenyl or 1-alkynyl halides. Tetrahedron Lett. 1979, 20, 3437-3440.

(15) Model compounds were employed where the diamine portion of the molecule was truncated leaving behind only the heterocyclic ring, primary carboxamide, and C-linked aryl substituent. To calculate potential energy surfaces, initial geometries were generated stepping through the two relevant dihedral angles at $\pm 0,5,10,30,60,90,120$, $150,170,175$, and 180 degrees. Dihedral angles of $(0,0)$ were defined to be the presumed planar bioactive conformation. Geometries were then optimized using GAUSSIAN 09 (rev. D01) at the B3LYP/6$31 \mathrm{G}^{*}$ level of theory keeping the two dihedral angles fixed at their initial values. Relative electronic energies across the geometries were then used to generate potential energy surface maps in MATLAB. Prediction of planar ground state geometries, such as for the compounds in Table 1, were performed similarly. Geometries of model compounds were generated through systematic enumeration of dihedral angles in 30 degree increments followed by unconstrained optimization. Compounds where the lowest-energy optimized structure was planar were considered for synthesis and experimental study.

(16) Predicted ground state geometry for the core of $\mathbf{8}$ depicting the pseudo 7-membered ring formed through intramolecular hydrogen bonding can be found in Figure S1 of the Supporting Information.

(17) Villasenor, A. G.; Kondru, R.; Ho, H.; Wang, S.; Papp, E.; Shaw, D.; Barnett, J. W.; Browner, M. F.; Kuglstatter, A. Structural insights for design of potent spleen tyrosine kinase inhibitors from crystallographic analysis of three inhibitor complexes. Chem. Biol. Drug Des. 2009, 73, 466-470.

(18) Liddle, J.; Atkinson, F. L.; Barker, M. D.; Carter, P. S.; Curtis, N. R.; Davis, R. P.; Douault, C.; Dickson, M. C.; Elwes, D.; Garton, N. S.; Gray, M.; Hayhow, T. G.; Hobbs, C. I.; Jones, E.; Leach, S.; Leavens, K.; Lewis, H. D.; McCleary, S.; Neu, M.; Patel, V. K.; Preston, A. G.; Ramirez-Molina, C.; Shipley, T. J.; Skone, P. A.; Smithers, N.; Somers, D. O.; Walker, A. L.; Watson, R. J.; Weingarten, G. G. Discovery of GSK143, a highly potent, selective and orally efficacious spleen tyrosine kinase inhibitor. Bioorg. Med. Chem. Lett. 2011, 21, 61886194.

(19) Thoma, G.; Smith, A. B.; van Eis, M. J.; Vangrevelinghe, E.; Blanz, J.; Aichholz, R.; Littlewood-Evans, A.; Lee, C. C.; Liu, H.; Zerwes, H. G. Discovery and profiling of a selective and efficacious syk inhibitor. J. Med. Chem. 2015, 58, 1950-1963.

(20) Lucas, M. C.; Bhagirath, N.; Chiao, E.; Goldstein, D. M.; Hermann, J. C.; Hsu, P.-Y.; Kirchner, S.; Kennedy-Smith, J. J.; Kuglstatter, A.; Lukacs, C.; Menke, J.; Niu, L.; Padilla, F.; Peng, Y.; Polonchuk, L.; Railkar, A.; Slade, M.; Soth, M.; Xu, D.; Yadava, P.; Yee, C.; Zhou, M.; Liao, C. Using ovality to predict nonmutagenic, orally efficacious pyridazine amides as cell specific spleen tyrosine kinase inhibitors. J. Med. Chem. 2014, 57, 2683-2691.

(21) A detailed account of the design considerations affording carboxamide 17 with a favorable in vitro, including mitigation of ion channel activity, and in vivo profile can be found in ref 12 . 